\title{
MONITORING OF LAND SURFACE DISPLACEMENTS WITHIN THE AREAS WITH INTENSIVE OIL PRODUCTION USING SATELLITE REMOTE SENSING DATA
}

\author{
Ilmir Nugmanov $^{(1)}$, Olga Chernova ${ }^{(2)}$ \\ ${ }^{(1)}$ Kazan Federal University, 18 Kremlyovskaya St., 420008, Kazan, Russian Federation, \\ Email:Ilmir.nugmanov@kpfu.ru \\ (2) Kazan Federal University, 18 Kremlyovskaya St., 420008, Kazan, Russian Federation, Email: re-sh@mail.ru
}

\begin{abstract}
The purpose of the study is to assess the informative value of differential synthetic aperture radar interferometry (DInSAR) as applied to estimation of modern crustal movements within the oil-producing regions of the Republic of Tatarstan (RT). For the first time in the RT, the earth's surface displacement maps were created using ERS and ALOS PALSAR data for different time periods in order to show possibility of using radar data to monitor urban areas. Interferometry results were then compared with geodetic leveling data. It is obvious that DInSAR resolution is incomparably greater than resolution of traditional methods used for monitoring of the earth's surface displacements. In the authors' opinion, the technology of using radar data to monitor the earth's surface displacements can become a principal approach to forecasting and prevention of industrial and natural disasters any time soon.
\end{abstract}

\section{INTRODUCTION}

The paper presents results of monitoring earth's surface displacements in the south-east of the Republic of Tatarstan, conducted on the basis of radar data obtained during the surveys of 1992-1995. The prospect of this kind of monitoring is obvious. Intensive oil production in the south-east of Tatarstan has led to the emergence of seismic activity and active vertical deformation processes. In this regard, in the 1980s a long-term program of environmental measures was deployed. It was aimed at rational development of hydrocarbon resources and provided environmental and industrial safety of the region. In 1991, the first study of modern deformation processes within the north-western part of the Romashkinskoye and Novo-Elhovskoe oilfields (areas of high seismic activity) was conducted by means of high-precision re-leveling. In the years since geodynamic research has covered the entire oil producing region of the south-eastern Tatarstan. Releveling provides valuable information, but it is also costly and time-consuming. Moreover, such an approach allows to obtain displacement values only at points of benchmarks, which it is not enough to get a complete picture. In recent times, an alternative method of earth's surface displacements estimation known as DlnSAR (differential synthetic aperture radar interferometry) has become widely accepted [1-4].
Effectiveness of this new method is explained by possibility to obtain the areal distribution of vertical displacement estimates, as well as by its independence of weather conditions. A huge number of DlnSAR projects covering the coal mines, large European cities [1], oilfields [2], areas of landslides [3] and earthquakes were reported to be successful. Despite the rapid development of this method, in Russia such studies are still extremely rare and, in most cases, are experimental. Therefore, the main objective of this work is to study the informativity of the remote sensing for purposes of monitoring and evaluation of modern crustal movements within the oil-producing areas of the Republic of Tatarstan.

\section{INPUT DATA}

In this project 49 ERS-1/2 descending SAR images were used. Also, the available ALOS PALSAR data were considered. Initial radar data covered several areas with highly developed oil-producing infrastructure (Almetyevsk, Zainsk, Sarmanovsk, Leninogorsk, Nizhnekamsk, Cheremshansk, Novosheshminsk).

Due to temporal and spatial decorrelation [2, 6], it was not possible to obtain a stable interferogram for the entire area (even in cases of small temporal baselines). As will soon be demonstrated, the most stable signals can be observed within urban areas. Despite the impressive collection of input data, only 10 interferograms were successfully generated: 7 for ERS$1 / 2$, and the other 3 for ALOS PALSAR. The area around the city of Almetyevsk was finally chosen to be studied in more detail.

\section{DATA PROCESSING}

Two images acquired by radar during its recurring passes over the same area are used for interferometric processing. Each pixel of the SAR image represents the coherent sum of the signal reflected from the point targets located on the surface within the resolution cell. Radar registers the received signal $S$ in a complex form, preserving the phase and the amplitude:

$$
S=A \exp (j \varphi) \exp (-j 4 \pi r / \lambda)
$$

being $A$ - the amplitude of the received signal; $\varphi-$ phase shift (due to reflection); $r$ - distance between the radar and the target; $\lambda$ - wavelength [6]. 
Interferometric processing is based on the phase difference between two reflected signals, which can be used to obtain the elevation of one reflecting element on the earth's surface relative to another. This information can be used in the further processing resulting in digital elevation model (DEM) and displacement maps. [4] $\varphi$ is a random component but it is excluded during the calculating. However, if time interval between recurring passages of the satellite is rather large, significant changes in land surface may occur and the remaining part of the $\varphi$ becomes the noise on the interferogram. Also if the baseline is too great, re-reflection phase is not excluded at all, and that leads to the destruction of the interferogram. Limitations of the interferometric processing are spatial and temporal baselines. [2] Spatial baseline (or just baseline) is the distance between the orbital positions of radar during its passes. Quality of the interferometry results depends on the perpendicular component of the baseline (for instance, the quality of displacement map decreases with increasing baseline). In the conditions of zero baseline interferogram contains only the phase difference. Thus, above a certain critical value of the baseline interferometric processing becomes simply impossible due to the spatial decorrelation.

The critical baseline $B_{n, c r}$ for each pair of images can be calculated using the formula shown in [2]. In the case of ERS-1 data, $B_{n, c r}$ was around $1000 \mathrm{~m}$. However, in this project the authors used pairs of images with baselines significantly lesser than $1000 \mathrm{~m}$.

Temporal baseline is a period of time between taking images that will make up the interferometric pair. This term is related to such an important issue as temporal decorrelation arising from changes in topography, vegetation , moisture, roughness and other properties of the reflecting surface that occurred during the period between surveys.

The problem of temporal decorrelation can be resolved by increasing the length of the signal wave (which increases the penetration power of waves) or reduction of the temporal baseline (i.e., time interval between two shots). Coherence value (from 0 to 100) represents the quality of the interferogram and is calculated as a correlation of two signals in complex form. It is also a measure of suitability of interferometric pair:

$$
\operatorname{Coh}=S_{1} S_{2}^{*} / \sqrt{\left(S_{1} S_{1}^{*} \bullet S_{2} S_{2}^{*}\right)}
$$

being $S_{1}, S_{2}$ - complex values of the reflected signal for the first (master) and the second (slave) images [5].

A comparative study of coherence maps shows extensive destruction of the interferometric phase because of the spatia1 and temporal decorrelation. Interferometric pairs with large spatial baselines are characterized by low coherency, and if the temporal baseline becomes greater than 1 year, the further processing of the pair will be impossible.

Comparison of coherence maps with geographical features of the area shows that the best targets for monitoring using radar sensing are urbanized territories. From this point of view, the most interesting object on the radar images in our case is the territory of Almetyevsk. Citizens have repeatedly experienced the effects of earthquakes, and there are hundreds of wells drilled around the city. It would seem that the city territory should undergo a systematic subsidence, but somewhat different results were obtained.

\section{RESULTS AND CONCLUSIONS}

Coherence maps were used to evaluate the interferometric pairs, and 4 of them were found to be suitable for displacement maps generation: 05.05.199214.07.1992; 05.05.1992-25.05.1993; 09.06.199203.08.1993; 05.05.1992-13.04.1995. The authors used SARScape software (an add-on for ENVI) for the interferometric processing. The processing algorithm itself is a set of standard procedures described in the manuals. [6]

Schemes of vertical displacements show that the territory of Almetyevsk in 1992 and 1993 experienced predominantly upward movements, with the most intense uplift along the linear zone approximately 1,5 $\mathrm{km}$ wide crossing the city in north-west direction. The displacement map obtained for the "09.06.199203.08.1993” pair shows lowering of the northern part of the city. At a later stage we can see lowering of both northern and southern parts of the city except of the already mentioned linear zone.

So, the displacements observed are not of linear character. Perhaps these movements have a periodic nature. Unfortunately, lack of data does not allow to evaluate its period and give a reasonable interpretation of this phenomenon. The average speed of vertical movements is about $5 \mathrm{~mm}$ per year according to the “05.05.1992-25.05.1993” pair.

Reliability of the data obtained by means of radar interferometry, as a rule, is verified by high-precision leveling or GPS monitoring. For this study the authors used results of leveling that was carried out in 19921995 within Almetyevsk geodynamic field.

The leveling network consisted of regional and local profiles. The leveling of first and second order was carried out with a mean square error of about $3 \mathrm{~mm}$ per $1 \mathrm{~km}$ of leveling line. Having no actual data in the form of value tables, the authors had to use the generalized results for geodetic observation cycles of 1992-1993 and 1993-1995. To compare displacement magnitudes obtained by means of interferometry and leveling, graphs of vertical displacements along the profiles A-A and B-B were plotted.

Graphs show rather good consistency of interferometry and leveling data sets. It is clear enough that resolution of the interferometry technique is much greater than resolution of the traditional method. Little differences in absolute values can be explained by inaccuracies of both methods and inaccuracies occurred during the process 
of positioning leveling chains relative to radar images. It is believed that the main reasons for the unsatisfactory DInSAR results are spatial and temporal decorrelation, which can be reduced through the use of higher resolution data obtained by the radars operating at much longer wavelengths.

In order to test this idea, several ALOS PALSAR images obtained for the south-eastern part of the RT were processed (spatial resolution - $20 \mathrm{~m}$, L-band). Further analysis and comparison with the ERS results showed that the coherence of interferometric images taken by ALOS is much higher. The coherence exceeds 0,5 along the major part of the territory covered by the image, whereas in case of ERS the coherence exceeds the minimum threshold only within the urban areas. This means that the results of interferometric processing of ALOS PALSAR data (i.e. vertical displacements values) can be considered reliable not only within the urbanized areas, but on the whole area of the research. In the absence of leveling data for the period of 2006 2007, the authors managed to estimate the accuracy of the DInSAR results by comparing the displacement maps generated for the periods from July to August, from August to October and from July to October. Algebraic addition of maps obtained for the first two periods gives a picture similar to that observed in the "overall" map created for the entire observation period (four months).

The graphs have the same shape but are offset relative to each other by $20 \mathrm{~mm}$. Possible cause for this may lie in the algorithm of interferometric processing - in particular, this shift can occur at the stage of surface slope correction, which consists in the following: earth's surface is tilted so as to form a right angle with the incident electromagnetic wave. Thus, getting rid of the calculation errors associated with non-vertical incidence of the wave, we get an error in the absolute values of vertical displacements.

So, as can be seen from the above, despite the spatial and temporal decorrelation (for which there is no universal cure) and experimental nature of the project, it is considered successful: a series of displacement maps obtained for the territory of Almetyevsk shows prospectivity of such monitoring, and medium resolution datasets (ERS, ENVISAT) can be used for reconnaissance, while for more accurate observations one should use higher resolution systems (TerraSAR-X) or systems with bigger wavelength (ALOS PALSAR).

This work was supported financially by the Ministry of Education and Science of the Russian Federation, contract no. 02.G25.31.0029.

1. Gabriel A. K., Goldstein R. M., Zebker H. A. (1989) Mapping small elevation changes over large areas: Differential radar interferometry. J. Geophys. Res. 94, 9183-9191.
2. Zeni G., Bonano M., Casu F. et al. (2011) Long-term deformation analysis of historical buildings through the advanced SBAS-DInSAR technique: The case study of the city of Rome, Italy. Journal of Geophysics and Engineering.8(3), 1-12.

3. Cascini L., Fornaro G., Peduto D. (2010) Advanced low- and full-resolution DInSAR map generation for slow-moving landslide analysis at different scales. Engineering Geology. 112(1-4), 29-42.

4. Rosen P. A., Hensley S., Joughin I. R. et al. (2000) Synthetic aperture radar interferometry. Proceeding of IEEE. 2000. 88(3), 333-380.

5. Richards M. A. (2007) Beginner's Guide to Interferometric SAR Concepts and Signal Processing. IEEE Aerospace and Electronic. 22(9), 5-22.

6. URL: http://www.sarmap.ch/pdf /SARGuidebook.pdf 